\title{
A deep-learning based raw waveform region-of-interest finder for the liquid argon time projection chamber (LArTPC)
}

Wanwei Wu' ${ }^{1}$, Lorenzo Uboldi², Michael Wang ${ }^{1}$, Tingjun Yang ${ }^{1}$ (on behalf of the ArgoNeuT collaboration) ${ }^{1}$ Fermilab, ${ }^{2}$ University of Milano

\section{Introduction}

- The LArTPC detector offers excellent spatial and energy resolution for the low energy physics.

- Extracting small signals from huge background becomes very challenging for low-energy phenomena.

- ArgoNeuT already shows the ability to reconstruct activity at the MeV scale in a LArTPC [1].

- An application of a 1D-CNN to the task of finding the region-of-interest $(\mathrm{ROI})$ in LArTPC raw waveforms is considered and tested on the ArgoNeuT experiment.

- The 1D-CNN ROI finder shows promise in its ability to extract small signals and can be implemented in early stages of reconstruction as a very effective filter to remove noise. It offers great potential for low-energy physics, such as supernova, solar neutrino interactions, and some new physics scenarios [1-2].

[1] Phys. Rev. $D 99$ (1),012002 (2019), arXiv: 1810.06502

[2] Phys. Rev. Letters 124, 131801 (2020) arXiv: 1911.07996

1D - Conventional Neural Network (CNN)

- This effort began by adapting 1D-CNNs used for analyzing cellphone accelerometer data to the analysis of LArTPC waveforms.

- After a successful proof-of-concept demonstration, the initial model was replaced with a more optimal design that was faster, more compact, and more accurate.

- Its capability as a simple classifier of signal vs noise waveforms was further expanded to permit the localization of the signal region within the waveform.
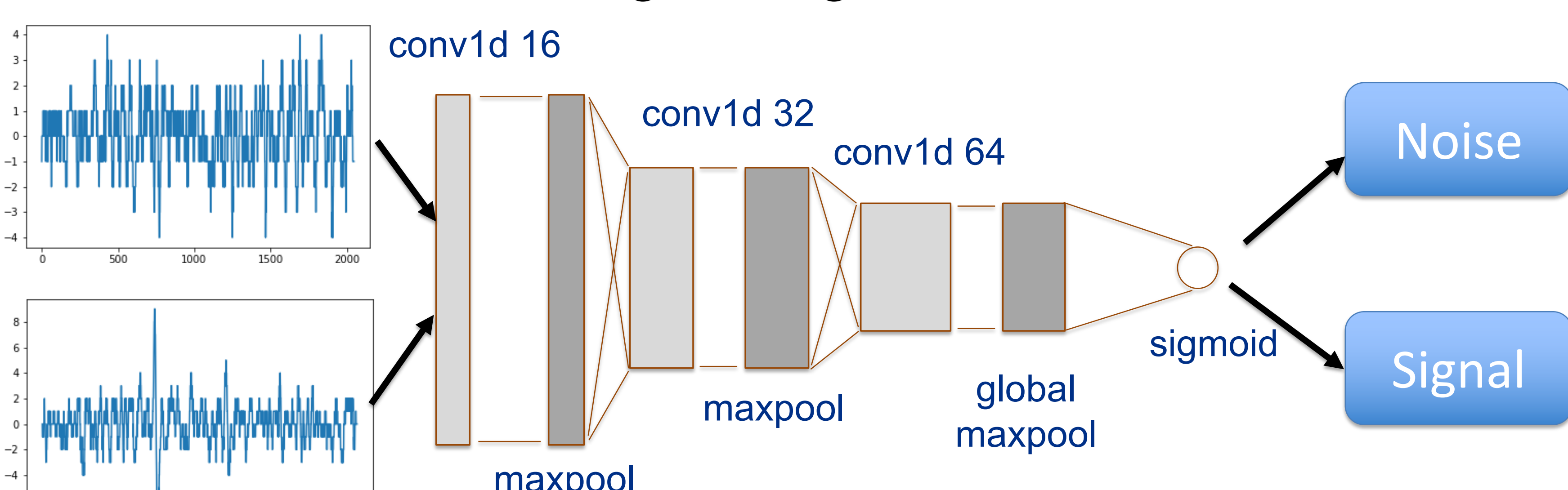

\section{ArgoNeuT LArTPC}

- Argon Neutrino Teststand detector located in front of MINOS near detector.

- $40 \times 47 \times 90 \mathrm{~cm}^{3}$ [vertical, drift, horizontal (beam)].

- 240 induction wires and 240 collection wires; 2048 samples with 198 ns sampling time.

- Data taking in $v / \bar{v}$ mode in 2009-2010.

\section{Data Driven Noise Model \& Noise Removal}

- Coherent noise removal was applied in order to study the data driven noise model.

- Perform an FFT on data noise and fit noise frequency to find noise model that can be used in simulation.

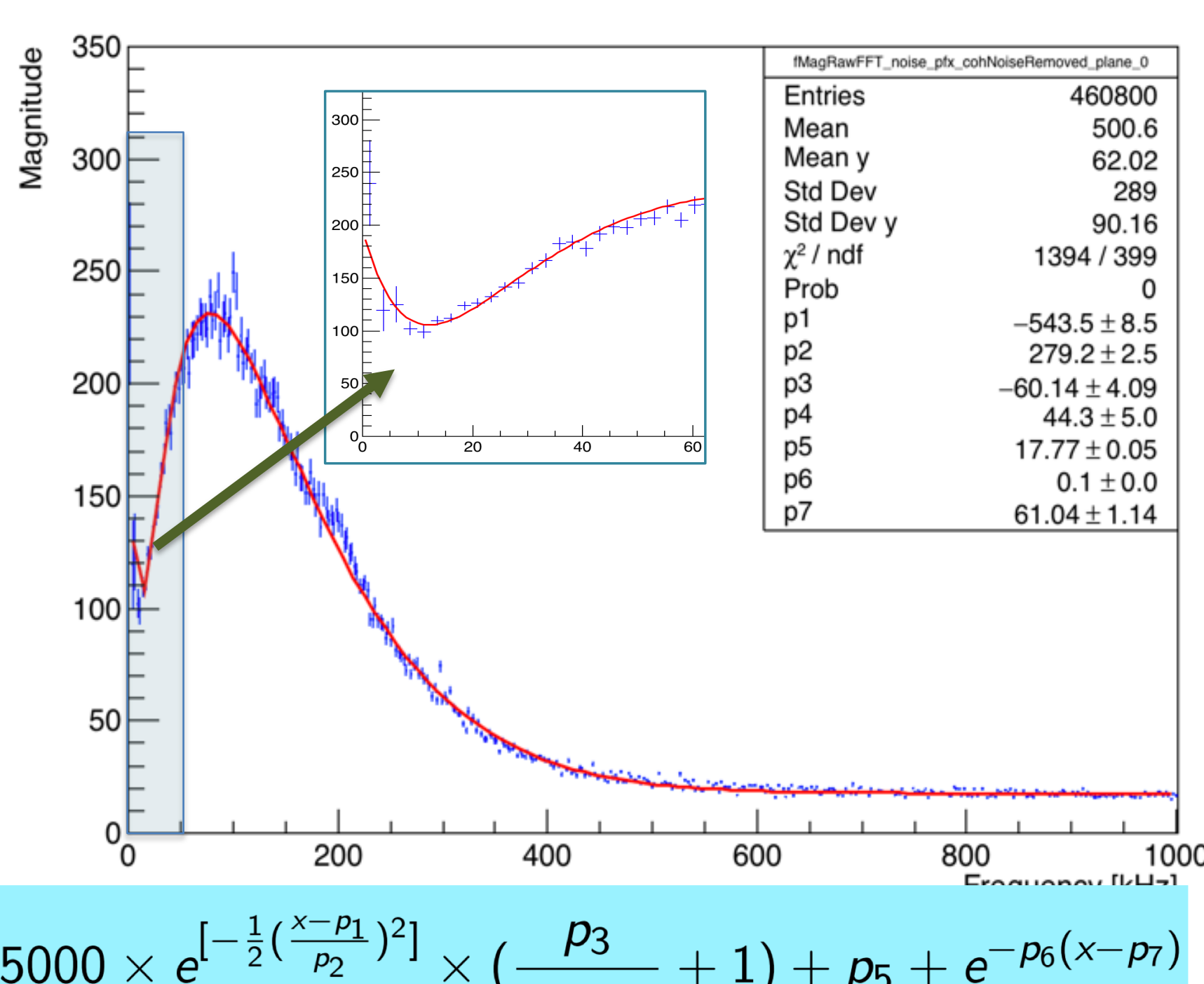

- Coherent noise removal is applied according to wire noise correlation.

- Adaptive baseline subtraction is used to remove noise from electronics and cable readout responses.

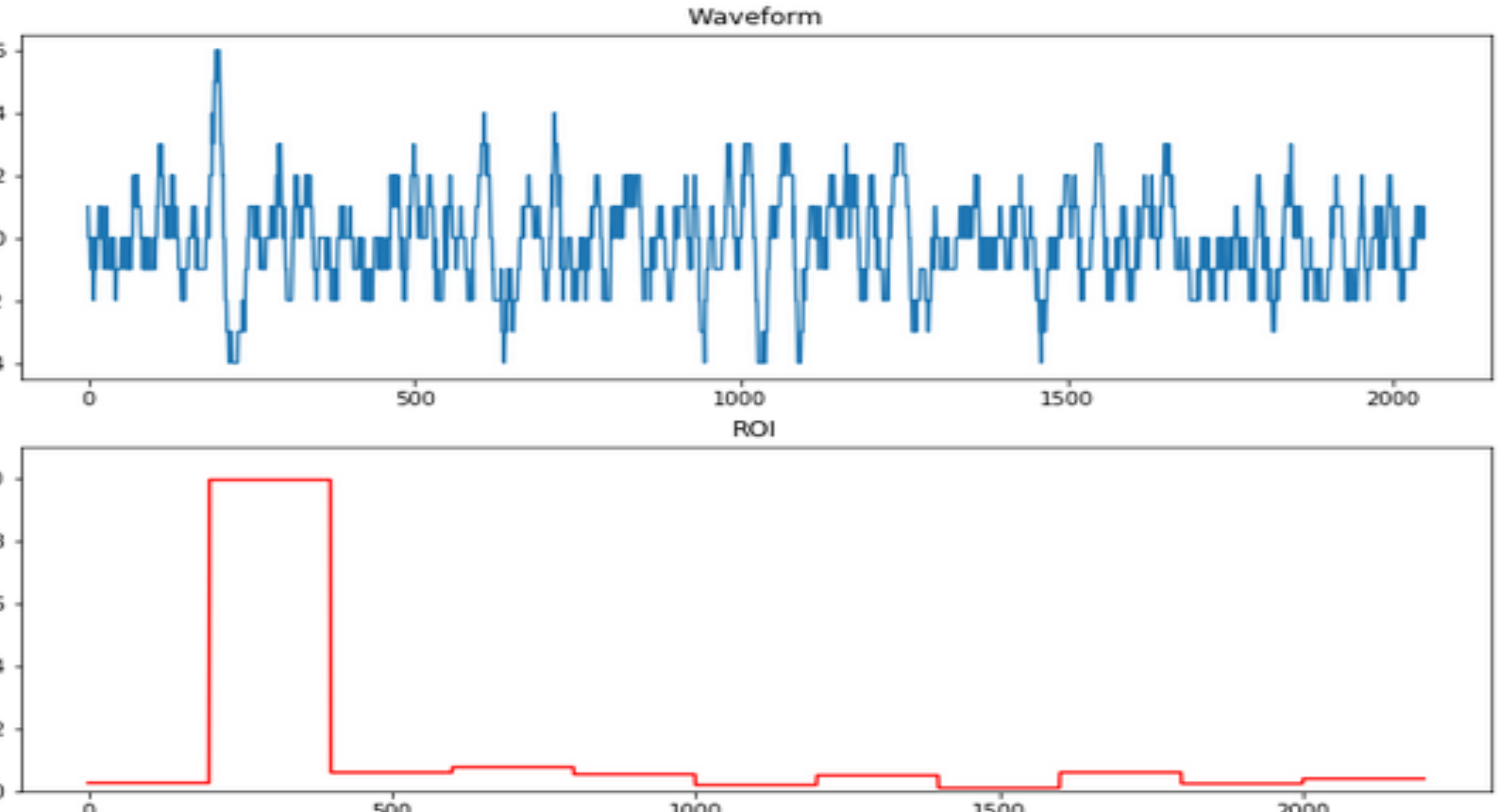

FERMILAB-POSTER-20-086-ND-SCD-V

\section{D-CNN ROI Finder}
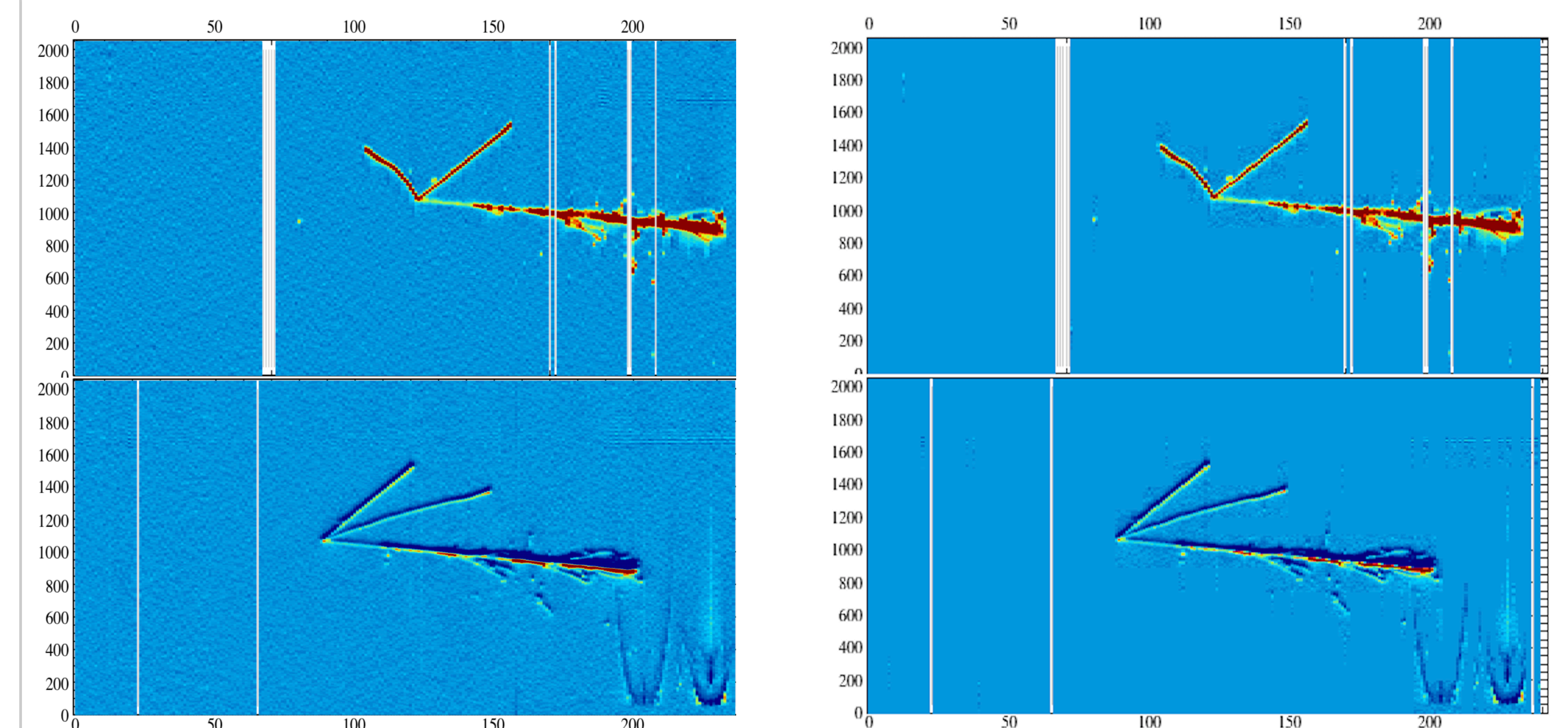

- Event displays for ArgoNeuT run 806, subrun 1, event 19509: left after noise removal and right from 1D-CNN ROI.

\section{Performance on Data and MC}

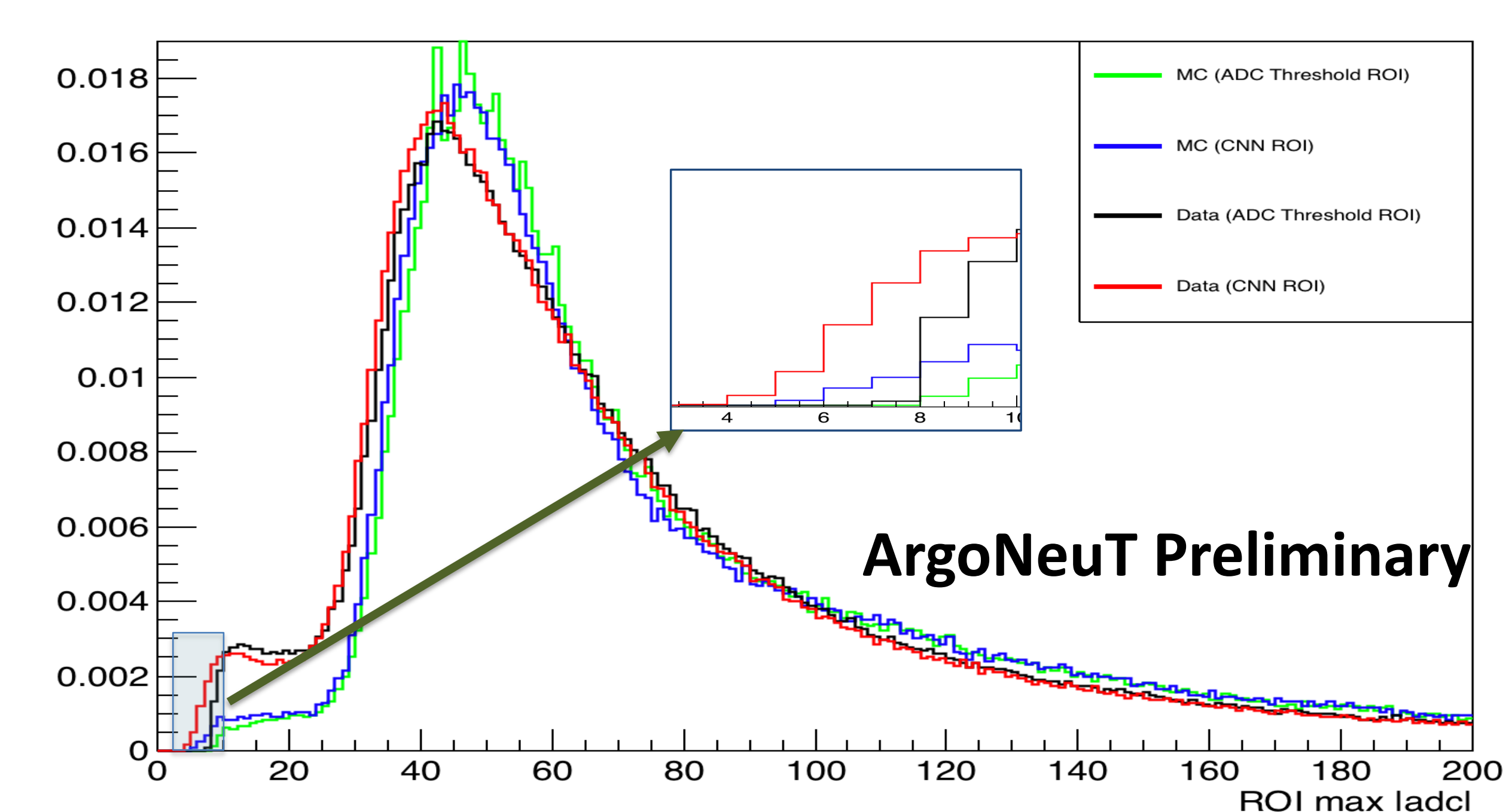

- Traditional over threshold ROI finder is considered in order to check the performance of 1D-CNN ROI finder.

- 1D-CNN ROI finder shows comparable results and demonstrates better sensitivity for small signals.

\section{Conclusions}

- Encouraging results in the application of a 1D-CNN to the task of finding ROI in raw LArTPC waveforms using ArgoNeuT data are shown.

- The 1D-CNN shows a promising ability to extract small signals and offers great potential for low-energy neutrino physics. 